International Mathematical Forum, Vol. 8, 2013, no. 11, 537 - 543 HIKARI Ltd, www.m-hikari.com

\title{
Existence of Positive Solutions of a Class of Discrete Difference Systems
}

\author{
Lihong Bai \\ Gansu Construction Vocational Technical College \\ Lanzhou, 730050, P.R. China \\ bailihong0305@126.com
}

Copyright (C) 2013 Lihong Bai. This is an open access article distributed under the Creative Commons Attribution License, which permits unrestricted use, distribution, and reproduction in any medium, provided the original work is properly cited.

\begin{abstract}
In this paper, we consider the existence of positive solutions of a class of discrete difference systems

$$
\begin{array}{ll}
-\Delta^{2} u(t-1)=\lambda f(v(t)), & t \in[1, T]_{\mathbb{Z}}, \\
-\Delta^{2} v(t-1)=\lambda g(u(t)), & t \in[1, T]_{\mathbb{Z}}, \\
u(0)=u(T+1)=0, & \\
v(0)=v(T+1)=0 &
\end{array}
$$

where $f, g \in C([0, \infty), \mathbb{R}), \lambda$ is a positive parameter. We prove the existence of a large positive solution for $\lambda$ large enough under suitable assumptions on $f$ and $g$. The proof of our main result is based upon the Schauder's fixed point theorem.
\end{abstract}

\section{Mathematics Subject Classification: 34B18}

Keywords: discrete difference systems; positive solutions; Existence; Schauder's fixed-point theorem 


\section{Introduction}

In this papers, we consider the existence of positive solutions of a class of discrete systems

$$
\begin{array}{ll}
-\Delta^{2} u(t-1)=\lambda f(v(t)), & t \in[1, T]_{\mathbb{Z}}, \\
-\Delta^{2} v(t-1)=\lambda g(u(t)), & t \in[1, T]_{\mathbb{Z}}, \\
u(0)=u(T+1)=0 & \\
v(0)=v(T+1)=0 &
\end{array}
$$

where $f, g \in C([0, \infty), \mathbb{R}), T>1$ is a fixed positive integer, $[1, T]_{\mathbb{Z}}:=\{1, \cdots, T\}$, $\lambda$ is a positive parameter.

Many problems in applied mathematics lead to the study of systems, see [1-7] and the references therein. Recently, there has been considerable interest in the existence of positive solutions of the discrete systems $[6,7]$.

In $[6,7]$, Sun and Li concerned with discrete system boundary value problems and gave some sufficient conditions for the existence of one or two positive solutions by using a nonlinear alternative of Leray-Schauder type and Krasnosel'skii's fixed point theorem in a cone.

However, to the best of our knowledge, there are very few works have been done to the existence of positive solutions of the discrete systems (1.1).

Our main features are as follows. First, (1.1) may be a semipositone problem, that is, $f$ and $g$ may take negative values. Second, we will study the systems (1.1) without imposing any sign conditions on $f(0), g(0)$ nor any monotonicity assumptions on $f, g$. Third, we will prove the existence of a large positive solution of (1.1) for $\lambda$ large. The arguments used were suggested by $[9]$.

In order to prove our main results, the following well-known fixed point theorems are needed.

Lemma 1.1.[10, 11] Let $C$ be a closed convex subset of the Banach space $E$. Suppose that $F: C \rightarrow C$ and $F$ is compact (i.e., bounded sets in $C$ are mapped into relatively compact sets). Then, $F$ has a fixed point in $C$.

Throughout the paper, we make the following assumptions:

(H1) $f, g \in C([0, \infty), \mathbb{R})$ and there exist positive numbers $L, K$ such that $f(x) \geq L, g(x) \geq L$ for $x \geq K$.

(H2) $\lim _{x \rightarrow \infty} \frac{f(M g(x))}{x}=0$ for every $M>0$.

By using Schauder's fixed point theorem, our main result is as follows.

Theorem 1.1. Let (H1), (H2) hold. Then there exists a positive number $\lambda^{*}$ such that (1.1) has a large positive solution $(u, v)$ for $\lambda \geq \lambda^{*}$. 
Remark 1.1. As an example, let $f(x)=x^{\sigma}-\varepsilon_{1}, g(x)=x^{\gamma}-\varepsilon_{2}$, where $\varepsilon_{1}, \varepsilon_{2}, \sigma, \gamma>0$ and $\sigma \gamma<1$. Then it is easily seen that $f, g$ satisfy assumptions (H1), (H2).

Remark 1.2. It is an interesting exercise in elementary analysis to show that assumption (H2) implies that

$$
\lim _{x \rightarrow \infty} \frac{g(M f(x))}{x}=0 \text { for every } M>0
$$

and hence both

$$
\lim _{x \rightarrow \infty} \frac{f(g(x))}{x}=0 \text { and } \lim _{x \rightarrow \infty} \frac{g(f(x))}{x}=0
$$

hold. However,

$$
\lim _{x \rightarrow \infty} \frac{f(g(x))}{x}=0
$$

does not imply that

$$
\lim _{x \rightarrow \infty} \frac{g(f(x))}{x}=0
$$

(for example, $f(x)=e^{x}$ and $g(x)=\frac{1}{2} \ln (1+x)$ ). Furthermore,

$$
\lim _{x \rightarrow \infty} \frac{f(g(x))}{x}=0 \text { and } \lim _{x \rightarrow \infty} \frac{g(f(x))}{x}=0
$$

do not imply that $\lim _{x \rightarrow \infty} \frac{f(M g(x))}{x}=0$ for every $M>0$, as the following example shows.

Let $a_{k}$ be an increasing sequence of positive numbers such that

$$
a_{1}=1, \lim _{k \rightarrow \infty} a_{k}=\infty \text { and } \frac{1+a_{k}}{1+a_{k+1}}<\frac{1}{k+1} \text { for } k \geq 1 .
$$

Let $g, f:[0, \infty) \rightarrow \mathbb{R}$ be such that $g=f, f(t)=1+a_{k}$ for $2+a_{k} \leq t \leq$ $1+a_{k+1}, k \geq 1, f$ is linear on $\left[1+a_{k}, 2+a_{k}\right], k \geq 2$, and $f$ is non-decreasing on $\left[0,2+a_{1}\right]$. Then it can be verified that $\lim _{t \rightarrow \infty} \frac{f(f(t))}{t}=0$, but since

$$
\frac{f\left(2\left(1+a_{k}\right)\right)}{1+a_{k}} \geq \frac{f\left(2+a_{k}\right)}{1+a_{k}}=1,
$$

it follows that $\frac{f(2 f(t))}{t} \nrightarrow 0$ as $t \rightarrow \infty$.

Remark 1.3. In the single-equation case,

$$
\begin{aligned}
& -\Delta^{2} \omega(t-1)=\lambda h(\omega), \quad t \in[1, T]_{\mathbb{Z}}, \\
& \omega(0)=\omega(T+1),
\end{aligned}
$$

and if $h$ is superlinear at $\infty$, then there are no positive solutions for $\lambda$ large unless $h(0)=h(1)=0$. However, here in the systems, even if one of the nonlinearities is superlinear at infinity, if the combined effect is sublinear , then positive solutions do exist for $\lambda$ large. 


\section{Proof of Theorem 1.1}

Let $f(x)=f(0)$ and $g(x)=g(0)$ for $x<0$. Let $\omega_{0}$ be the solution of

$$
\begin{aligned}
& -\Delta^{2} \omega(t-1)=1, \quad t \in[1, T]_{\mathbb{Z}} \\
& \omega_{0}(0)=\omega_{0}(T+1)
\end{aligned}
$$

Note that $\omega_{0}(t)=\frac{1}{2} t(T+1-t)$, with $w_{0}(t)>0, t \in[1, T]_{\mathbb{Z}}$. Let

$$
X=\left\{u \mid u:[0, T]_{\mathbb{Z}} \rightarrow \mathbb{R}, u(0)=u(T+1)=0\right\},
$$

then $X$ is a Banach space with the norm $\|u\|=\max _{t \in[0, T+1]_{\mathbb{Z}}}|u(t)|$, and $A: X \times$ $X \rightarrow X \times X$ be defined by

$$
A(u, v)=\left(\lambda \sum_{i=1}^{T} G(t, i) f(v(i)), \lambda \sum_{i=1}^{T} G(t, i) g(u(i))\right)
$$

where

$$
G(t, i)=\left\{\begin{array}{lc}
\frac{(T+1-t) i}{T+1}, & 1 \leq i \leq t \leq T+1 \\
\frac{(T+1-i) t}{T+1}, & 0 \leq t \leq i \leq T
\end{array}\right.
$$

is the Green's function of

$$
\begin{aligned}
& -\Delta z^{2}(t-1)=0, t \in[1, T]_{\mathbb{Z}} \\
& z(0)=z(T+1)=0
\end{aligned}
$$

Then $A$ is completely continuous and fixed points of $A$ are solutions to problem (1.1). Let $\psi=\left(\omega_{\lambda}, \omega_{\lambda}\right)$, where $\omega_{\lambda}=\frac{1}{2} \lambda L \omega_{0}$, and $\phi=\left(C_{\lambda} \omega_{0}, \lambda \tilde{g}\left(C_{\lambda} \delta\right) \omega_{0}\right)$, where $\delta=\left\|\omega_{0}\right\|, \tilde{g}(x)=\sup _{y \leq x} g(y)$ and $C_{\lambda}$ is large enough so that $\psi \leq \phi$. We claim that $A:[\psi, \phi] \rightarrow[\psi, \phi]$ for $\lambda$ is sufficiently large. Once the claim is proved, it then follows from the Schauder's fixed point theorem that $A$ has a fixed point $(u, v)$ with $u \geq w_{\lambda}$ and $v \geq w_{\lambda}$. Clearly, $u(t), v(t) \rightarrow \infty$ uniformly on $[0, T+1]_{\mathbb{Z}}$ as $\lambda \rightarrow \infty$. To prove the claim, we will verify the following:

(A) If $(u, v) \geq \psi$, then $A(u, v) \geq \psi$.

(B) If $(u, v) \leq \phi$, then $A(u, v) \leq \phi$.

Proof of A. Let $(u, v) \geq \psi$, we shall verify that

$$
\sum_{i=1}^{T} G(t, i) f(v(i)) \geq \frac{1}{2} L \sum_{i=1}^{T} G(t, i)
$$


Now, since $u, v \geq \omega_{\lambda}$, we have $u, v \geq K$ if $\lambda$ is sufficiently large, and hence $f(v) \geq L$. Consequently,

$$
\begin{aligned}
\sum_{i=1}^{T} G(t, i) & f(v(i))-\frac{1}{2} L \sum_{i=1}^{T} G(t, i) \\
& =\sum_{i=1}^{T} G(t, s)\left(f\left(v(i)-\frac{1}{2} L\right)\right. \\
& \geq \frac{1}{2} L w_{0} \\
& \geq 0 .
\end{aligned}
$$

By using the same argument, we obtain

$$
\sum_{i=1}^{T} G(t, i) g(u(i)) \geq \frac{1}{2} L \sum_{i=1}^{T} G(t, i)
$$

for $\lambda$ large.

Proof of B. Let $(u, v) \leq \phi=\left(C_{\lambda} \omega_{0}, \lambda \tilde{g}\left(C_{\lambda} \delta\right) \omega_{0}\right)$. We shall verify that $A(u, v) \leq \phi$ or

$$
\lambda \sum_{i=1}^{T} G(t, i) f(v(i)) \leq C_{\lambda} \omega_{0}
$$

and

$$
\lambda \sum_{i=1}^{T} G(t, i) g(u(i)) \leq \lambda \tilde{g}\left(C_{\lambda} \delta\right) \omega_{0} .
$$

Now, by (H2), it is an interesting exercise in elementary analysis to show that $\lim _{x \rightarrow \infty} \tilde{f}(M \tilde{g}(x)) / x=0$ for each $M>0$. Hence we have

$$
\begin{aligned}
\lambda \sum_{i=1}^{T} G(t, i) f(v(i)) & \leq \lambda \sum_{i=1}^{T} G(t, i) \tilde{f}\left(\lambda \tilde{g}\left(C_{\lambda} \delta\right) \omega_{0}\right) \\
& \leq \lambda \sum_{i=1}^{T} G(t, i) \tilde{f}\left(\lambda \tilde{g}\left(C_{\lambda} \delta\right) \delta\right) \\
& =\lambda \tilde{f}\left(\lambda \delta \tilde{g}\left(C_{\lambda} \delta\right)\right) \omega_{0} \\
& \leq C_{\lambda} \omega_{0}
\end{aligned}
$$

if $C_{\lambda}$ is large enough, proving (B1). Next, since $u(i) \leq C_{\lambda} \delta$, we obtain

$$
\lambda \sum_{i=1}^{T} g(t, i) g(u(i)) \leq \lambda \tilde{g}\left(C_{\lambda} \delta\right) \omega_{0},
$$


which is (B2), and the claim is proved. This completes the proof of theorem1.1.

Remark 2.1. Using similar arguments as in the proof of theorem 1.1, it can be shown that the system

$$
\begin{array}{ll}
-\Delta^{2} u(t-1)=\lambda f(u, v), & t \in[1, T]_{\mathbb{Z},}, \\
-\Delta^{2} v(t-1)=\lambda g(u . v), & t \in[1, T]_{\mathbb{Z}}, \\
u(0)=u(T+1)=0, & \\
v(0)=v(T+1)=0 &
\end{array}
$$

has a positive solution for $\lambda$ large when $f, g$ satisfy the following conditions. $(\mathrm{H} 1)^{\prime} f, g \in C([0, \infty) \times[0, \infty), \mathbb{R})$ and there exist positive numbers $L, K$ such that $f(x, y) \geq L, g(x, y) \geq L$ for $x \geq K, y \geq K$.

$(\mathrm{H} 2)^{\prime}$ For each $M>0$,

$$
\lim _{x \rightarrow \infty} \frac{\tilde{f}(x, M \tilde{g}(x, x))}{x}=0 \text { and } \frac{\tilde{g}(x, M \tilde{g}(x, x))}{\tilde{g}(x, x)} \leq 1 \text { for large } x,
$$

where $\tilde{h}(u, v)=\sup _{x \leq u, y \leq v} h(x, y)$.

Note that these conditions are satisfied if $f(u, v)=u^{\alpha}+v^{\beta}-\epsilon_{1}$ and $g(u, v)=$ $u^{\gamma}+v^{\sigma}-\epsilon_{2}$, where $\alpha, \gamma, \sigma, \beta \sigma, \beta \gamma \in(0,1)$. In particular, $\beta$ could be more than 1 .

\section{REFERENCES}

[1] P.J.Y. Wong, Solutions of constant signs of a system of SturmCLiouville boundary value problems, Math. Comput. Model. 29 (1999) 27-38.

[2] Y.M. Shi, S.Z. Chen, Spectral theory of second order vector difference equations, J. Math. Anal. Appl. 239 (1999) 195-212.

[3] G.R. Gavalas, Nonlinear Differential Equations of Chemically Reacting Systems, Springer- Verlag, New York, 1968.

[4] A.W. Leung, Systems of Nonlinear Partial Differential Equations, Kluwer Academic Publishers, Dordrecht, The Netherlands, 1989.

[5] K.L. Cooke, J.L. Kaplan, A periodic threshold theorem for epidemics and population growth, Math. Biosci. 31 (1976) 87-104.

[6] J.P. Sun, W.T. Li, Multiple positive solutions of a discrete difference system, Appl. Math. Comp. 143 (2003) 213-221.

[7] J.P. Sun, W.T. Li, Existence of positive solutions of boundary value problem for a discrete difference system, Appl. Math. Comp. 156 (2004) 857-870. 
[8] D. D. Hai, R. Shivaji, An existence result on positive solutions for a class of semilinear elliptic systems, Proc. Roy. Soc. Edinburgh Sect. A 134 (2004) 137-141.

[9] K. Deimling, Nonlinear Functional Analysis, New York, Springer-Verlag, 1985 .

[10] M. Krasnosel'skii, Positive Solutions of Operator Equations, Noordhoff, Groningen, 1964.

Received: November, 2012 